\title{
Sonication of Kirschner wire as a tool for the microbiological diagnosis of diabetic foot infection - preliminary results
}

\author{
Rodrigo Paes Leme, MD ${ }^{1,2 *}$, Jéssica Chaves ${ }^{3}$, Luiz Carlos Gonçalves, MD ${ }^{4}$, Leonardo César Alvim, MD ${ }^{4}$, João \\ Roberto de Almeida, MD ${ }^{4}$, Ana Cláudia de Abreu 5 , Leonardo Renó, MD ${ }^{4,6}$
}

1 Department of Infectious Diseases, Hospital Unimed Volta Redonda, Volta Redonda - Rio de Janeiro - Brasil; rodrigo.cpaesleme@gmail.com

2 PhD student, Laboratório Especial de Microbiologia Clínica (LEMC), Universidade Federal de São Paulo, Escola Paulista de Medicina, São Paulo - SP, Brasil

3 Laboratory of Microbiology, Hospital Unimed Volta Redonda, Volta Redonda - Rio de Janeiro - Brasil jessica.chaves@unimedvr.com.br

4 Department of Vascular Surgery, Hospital Unimed Volta Redonda, Volta Redonda - Rio de Janeiro - Brasil; c.t.v.r@hotmail.com

5 Biostatistics, member of the Conselho Regional de Estatística de Minas Gerais, Belo Horizonte - Minas Gerais - Brasil

6 PhD student, Universidade de São Paulo, São Paulo - SP, Brasil

* Correspondence; rodrigo.cpaesleme@gmail.com Tel.: +55 24998492092

\section{Abstract:}

Background: Diabetic foot infection (DFI) is the commonest diabetic problem requiring hospital admission. Culture yield can be challenging, particularly in the presence of biofilms. Literature confirms biofilms are ubiquitous in diabetic foot ulcer, although, there is not a microbiologic diagnostic approach regarding biofilm disruption on DFI. We postulated sonicating a stainless-steel wire along with tissue samples into the thioglycollate broth media (TBM) may improve the diagnosis of DFI. Method: Prospective unicentric study that assessed patients with DFI who underwent surgical debridement. The vascular surgery team collected tissue fragments and inoculated the specimens into three TBM to execute the conventional culture method (CCM), and additional fragments to place into other TBM along with a Kirschner wire (K-wire - Kw method). The microbiologist processed the samples and the resultant sonication fluid in aerobic sheep-blood agar after 24 hours, 5 and 10 days of incubation. Both methods were compared (Wilcoxon test; $\mathrm{p}<$ 0.05). Results: The number of pathogens isolated in each method was not statistically significant ( $\mathrm{p}$ $=0.414): \mathrm{CM}=1.67$ ( \pm 0.92$) ; \mathrm{KwM}=1.75$ ( \pm 0.94$)$. The KwM was not inferior to CCM. In addition, despite the absence of statistical significance, the KwM detected more pathogens than CCM.

Keywords: Diabetic foot infection; biofilm disruption; Kirschner wire; sonication 


\section{Introduction}

DFI is the most familiar diabetic problem requiring hospital admission and a major part of the amount of work of clinical specialists. Besides, DFIs are associated with substantial morbidity, mortality, and reduced quality of life. Early suspicion diagnosis is essential to improve outcomes [1]. The microbiology of DFI modifies by characteristics of the patient (e.g., previous antibiotic course, recent hospitalisation) as well as the severity of disease. Minor DFI tend to be caused by Gram-positive cocci, and moderate DFI by Gram-positive and Gramnegative pathogens. In severe DFI, the infection can be polymicrobial, concerning Gram-positive and Gramnegative bacteria along with Candida spp. [1]. Obtaining a specimen for culture provides valuable information on the causative pathogen(s) along with their antibiotic susceptibility, allowing appropriate selection of antibiotic therapy. Though, culture yield can be challenging, particularly in the presence of biofilms [2]. Rising evidence surrounded by the literature confirms biofilms are ubiquitous in diabetic foot ulcer (DFU) and suggested that they participate to delayed wound healing [3]. Johani et al. investigated the presence of biofilm in DFU applying microscopy combined with molecular approaches. All 65 DFU specimens evaluated by microscopy contained biofilm, $(\mathrm{P}<0.001)$. The researchers detected the existence of mono and multi-species biofilms in the same tissue segments, and when DNA sequencing analysis showed varied polymicrobial communities [4]. The consequences of harboring biofilms are negative, since it increases the chances of therapeutic failure while its complex structure hinder immune action, antimicrobial penetration, and wound healing. Therefore, the biofilm disruption may be crucial to obtain better outcomes, since it could allow the identification sessile pathogenic microorganisms. Until now, there is not a microbiologic diagnostic approach regarding biofilm disruption on DFI. Here, we postulated that sonication of a stainless steel wire along with tissue samples into the TBM could disrupt the biofilm, allowing an improvement on microbiological diagnosis of DFI. In the background, we aimed to evaluate the risk factors for amputation.

\section{Materials and Methods}

We performed a prospective unicentric study that assessed patients with DFI who underwent surgical debridement between April 2018 and April 2021 at a 250-bed tertiary hospital centre. The vascular surgery team collected three fragments of soft tissue or bone then inoculated the specimens into three TBM to posterior execution of the conventional culture method (CCM), and extra three fragments along with a sterile Kirschner wire (K-wire) gaging $5 \mathrm{~cm}$ were inoculated together into other three TBM ( $\mathrm{Kw}$ method). Each TBM received only 67 one specimen. The TBM harboring a K-wire was vortexed for 30 seconds then sonicated for 1 minute, and vortexed (30 seconds) again as soon as they arrived at the laboratory, on the fifth and tenth days of incubation at 35 to $37^{\circ} \mathrm{C}$ in $5 \%$ to $7 \% \mathrm{CO} 2$. We sonicated earlier since distinct bacteria could be attached to surfaces for seconds 
a K-wire) in aerobic sheep-blood agar plates at $24 \mathrm{~h}, 5$ and 10 days. We do not proceed with anaerobic culture. 73 The microorganisms were identified by means of the VITEK 2 system. Both methods were compared (Wilcoxon 74 test). In addition, we applied the Mann-Whitney test to assess whether two independent samples were taken 75 from populations with equal means. All results were considered significant for a probability of significance of 76 less than $5 \%(p<0.05)$, thus having at least $95 \%$ confidence in the conclusions presented. 77

3. Results

Overall, twenty-four patients were enrolled, 29.2\% female and 70.8\% male. The patient's age ranged from 33 to 86 years (57.8 \pm 12.2$) ; 58.3 \%$ and $41.7 \%$ were PEDIS 3 and 4 , respectively. The number of pathogens isolated in each method was not statistically significant $(\mathrm{p}=0.414)$ : $\mathrm{CMC}=1.67( \pm 0.92) ; \mathrm{KwM}=1.75( \pm 0.94)($ Table 1$)$. 84 There was a microbiological agreement in $75 \%$ of the situations (18/24 cases) concerning the two methods. The CCM detected an additional pathogen in two different cases, (Pseudomonas aeruginosa and Enterococcus faecalis). 86 The KwM identified an extra pathogen in four patients: Klebsiella pneumoniae and Proteus mirabilis (in the same 87 case); P. aeruginosa, Morganella morganii and Streptococcus agalactiae in three different cases. Table 2 shows the 88 association between the need for amputation and the factors of interest. There was a significant association 89 between amputation and hospitalisation in the last six months $(p=0,037)$. All patients in which amputation was 90 performed were not hospitalized in the last six months, against $38.9 \%$ in the group where amputation was not 91 performed.

Table 1. Characterization of patients regarding the number of microorganisms considering the method.

\begin{tabular}{c|c|c|c}
\hline \multirow{2}{*}{ Method } & \multicolumn{2}{|c|}{ Descriptive measures } & \multirow{2}{*}{$\boldsymbol{P}$} \\
\cline { 2 - 3 } & Minimum-Maximum & Average + SD ${ }^{3}$ & \\
\hline CCM $^{1}$ & $0,0-4,0$ & $1,67 \pm 0,92$ & 0,414 \\
KwM $^{2}$ & $0,0-4,0$ & $1,75 \pm 0,94$ & \\
\hline
\end{tabular}

${ }^{1}$ Conventional conventional method 
Table 2. The association between amputation and the variables of interest.

\begin{tabular}{|c|c|c|c|}
\hline \multirow{2}{*}{ Variables } & \multicolumn{2}{|c|}{ Amputation } & \multirow{2}{*}{$p$} \\
\hline & No & Yes & \\
\hline \multicolumn{4}{|l|}{ Sex } \\
\hline Female & $12(66,7 \%)$ & $4(80 \%)$ & \multirow{2}{*}{$1,000^{1}$} \\
\hline Male & $6(33,3 \%)$ & $1(20 \%)$ & \\
\hline Age & $57,4 \pm 12,9$ & $58,8 \pm 11,9$ & \multirow[b]{2}{*}{0,801} \\
\hline $\mathrm{P}_{50}\left(\mathrm{P}_{25}-\mathrm{P}_{75}\right)$ & $57,5(46,8-64,3)$ & $55,0(49,0-70,5)$ & \\
\hline \multicolumn{4}{|l|}{ PEDIS severity } \\
\hline PEDIS 3 & $11(61,1 \%)$ & $2(40 \%)$ & \multirow{2}{*}{$0,618^{1}$} \\
\hline PEDIS 4 & $7(38,9 \%)$ & $3(60 \%)$ & \\
\hline
\end{tabular}

Hemoglobin (g/dL)

\begin{tabular}{|c|c|c|}
\hline Average \pm SD & $9,6 \pm 2,1$ & $10,2 \pm 1,5$ \\
\hline $\mathrm{P}_{50}\left(\mathrm{P}_{25}-\mathrm{P}_{75}\right)$ & $9,0(8,0-11,0)$ & $10,0(9,0-11,5)$ \\
\hline
\end{tabular}

Antibiotic use us last 3 months

$\begin{array}{lccc}\text { No } & 4(22,2 \%) & 1(20 \%) & 1,000^{1} \\ \text { Yes } & 14(77,8 \%) & 4(80 \%) & \end{array}$

Hospitalisation in the last 6 months

\begin{tabular}{|c|c|c|c|}
\hline No & $7(38,9 \%)$ & $5(100 \%)$ & \\
\hline Yes & $11(61,1 \%)$ & $0(0 \%)$ & \\
\hline
\end{tabular}

Use of $\mathrm{CIP}^{3}$ or LEV ${ }^{4}$ in the last 3 months

$\begin{array}{ccc}\text { No } & 8(44,4 \%) & 3(60 \%) \\ \text { Yes } & 4(22,2 \%) & 2(40 \%) \\ \text { Unknown } & 6(33,3 \%) & 0(0 \%)\end{array}$

\section{Gram-negative}

$\begin{array}{lccc}\text { No } & 12(66,7 \%) & 4(80 \%) & 1,000^{1} \\ \text { Yes } & 6(33,3 \%) & 1(20 \%) & \end{array}$




\section{Osteomyelitis}

$\begin{array}{lcc}\text { No } & 11(61,1 \%) & 1(20 \%) \\ \text { Yes } & 7(38,9 \%) & 4(80 \%)\end{array}$

${ }^{1}$ The probability of significance refers to Fisher's exact test.

${ }^{2}$ The probability of significance refers to the Mann-Whitney test.

${ }^{3}$ Ciprofloxacin

${ }^{4}$ Levofloxacin

\section{Discussion}

Despite the absence of statistical significance, it is important to consider the low sampling and the absence of a sub-analysis of patients with chronic diabetic foot infection (so far, 17 patients), often harboring extensive, polymicrobial and mature biofilms [4-11]. In addition, we do not perform anaerobic culture due to the insufficiency of the essential supplies. It is possible to obtain expressive results from the statistical point of view with a larger sampling, which allows the sub analysis of chronic infections and anaerobic cultivation. There are many questions still unanswered, such as (1) the ideal media to insert the tissue samples, (2) which material composition is most appropriate to incubate along with the tissues - stainless steel, silicone, or polyurethane, for example, (3) the number of samples to take in, and (4) the impact of early sonication as well as the instances chose to sonicate. It must not be forgotten that we intend an early diagnosis, which positively impacts treatment, for cure or disease-free survival reasons.

We do not know certainly why the association between amputation and the absence of previous

The KwM may be a useful microbiologic diagnostic tool to complement the conventional culture method 
participants, and anaerobic culture application. Not less crucial are the questions regarding the ideal media, type 127 of material composition, the optimal number of tissue samples, and the ideal moments to sonicate.

Author Contributions: Conceptualization, Rodrigo Cuiabano Paes Leme; methodology, Rodrigo Cuiabano Paes Leme, Jéssica

Chaves and Leonardo Renó; software, Rodrigo Cuiabano Paes Leme.; validation, Luiz Carlos Gonçalves, Leonardo César Alvim resources, Rodrigo Cuiabano Paes Leme, Jéssica Chaves, and Leonardo Renó; data curation, Rodrigo Cuiabano Paes Leme; writing - original draft preparation, Rodrigo Cuiabano Paes Leme; writing-review and editing, Rodrigo Cuiabano Paes Leme,

Funding: This research received no external funding.

Institutional Review Board Statement: Ethical review and approval were waived for this study, due to following reasons:

1. because it is an observational and prospective study, which will employ mostly information from medical records, institutional

2. because all data was carried out and analyzed anonymously,

3. because the results resulting from the study will be presented in aggregate form, not allowing the individual identification of the participants, and

4. because it is a non-interventional study and without alterations or influences in the routine and treatment of the research participant, and consequently without adding risks or damage to their well-being.

Informed Consent Statement: Ethical review and approval were waived for this study. The reasons are explained on 148

"Institutional Review Board Statement".

Data Availability Statement: Data available on https://figshare.com/

Conflicts of Interest: The authors declare no conflict of interest. 
1. Saeed, K.; Esposito, S.; Akram, A.; Ascione, T.; Bal, A.M.; Bassetti, M.; Carnelutti, A.; Chan, M.; Davis, J.; Dryden, M.; et al. Hot topics in diabetic foot infection. Int. J. Antimicrob. Agents 2020, 55, 105942, doi:10.1016/j.ijantimicag.2020.105942.

2. Lipsky, B.A. Stopping Antibiotic Therapy for a Diabetic Foot Infection. Int. J. Low. Extrem. Wounds 2015, 14, 307-308, doi:10.1177/1534734615607145.

3. Lipsky, B.A.; Senneville, É.; Abbas, Z.G.; Aragón-Sánchez, J.; Diggle, M.; Embil, J.M.; Kono, S.; Lavery, L.A.; Malone, M.; 166 Asten, S.A.; et al. Guidelines on the diagnosis and treatment of foot infection in persons with diabetes (IWGDF 2019 update). 167 Diabetes. Metab. Res. Rev. 2020, 36, doi:10.1002/dmrr.3280.

4. Johani, K.; Malone, M.; Jensen, S.; Gosbell, I.; Dickson, H.; Hu, H.; Vickery, K. Microscopy visualisation confirms multispecies biofilms are ubiquitous in diabetic foot ulcers. Int. Wound J. 2017, 14, 1160-1169, doi:10.1111/iwj.12777.

5. Pouget, C.; Dunyach-Remy, C.; Pantel, A.; Schuldiner, S.; Sotto, A.; Lavigne, J.P. Biofilms in diabetic foot ulcers: Significance and clinical relevance. Microorganisms 2020, 8, 1-15.

6. Armstrong, D.G.; Boulton, A.J.M.; Bus, S.A. Diabetic Foot Ulcers and Their Recurrence. N. Engl. J. Med. 2017, 376, 2367-2375, doi:10.1056/nejmra1615439.

169

7. Dowd, S.E.; Wolcott, R.D.; Sun, Y.; McKeehan, T.; Smith, E.; Rhoads, D. Polymicrobial nature of chronic diabetic foot ulcer biofilm infections determined using bacterial tag encoded FLX amplicon pyrosequencing (bTEFAP). PLoS One 2008, 3, 176 doi:10.1371/journal.pone.0003326.

8. Redel, H.; Gao, Z.; Li, H.; Alekseyenko, A. V.; Zhou, Y.; Perez-Perez, G.I.; Weinstock, G.; Sodergren, E.; Blaser, M.J. 178 Quantitation and composition of cutaneous microbiota in diabetic and nondiabetic men. J. Infect. Dis. 2013, 207, 1105-1114, 179 doi:10.1093/infdis/jit005.

9. Gontcharova, V. A Comparison of Bacterial Composition in Diabetic Ulcers and Contralateral Intact Skin. Open Microbiol. J. 181 2010, 4, 8-19, doi:10.2174/1874285801004010008.

10. Jneid, J.; Cassir, N.; Schuldiner, S.; Jourdan, N.; Sotto, A.; Lavigne, J.-P.; La Scola, B. Exploring the Microbiota of Diabetic Foot Infections With Culturomics. Front. Cell. Infect. Microbiol. 2018, 8, doi:10.3389/fcimb.2018.00282.

11. Pereira, S.G.; Moura, J.; Carvalho, E.; Empadinhas, N. Microbiota of chronic diabetic wounds: Ecology, impact, and potential for innovative treatment strategies. Front. Microbiol. 2017, 8.

12. Sen, P.; Demirdal, T.; Emir, B. Meta-analysis of risk factors for amputation in diabetic foot infections. Diabetes. Metab. Res. Rev. 2019, 35, doi:10.1002/dmrr.3165. et al. Predictors of lower-extremity amputation in patients with an infected diabetic foot ulcer. Diabetes Care 2015, 38, 852857, doi:10.2337/dc14-1598.

14. Uysal, S.; Arda, B.; Taşbakan, M.I.; Çetinkalp, Ş.; Şimşir, I.Y.; Öztürk, A.M.; Uysal, A.; Ertam, İ. Risk factors for amputation in patients with diabetic foot infection: a prospective study. Int. Wound J. 2017, 14, 1219-1224, doi:10.1111/iwj.12788.

15. Lu, Q.; Wang, J.; Wei, X.; Wang, G.; Xu, Y. Risk Factors for Major Amputation in Diabetic Foot Ulcer Patients. Diabetes. Metab. Syndr. Obes. 2021, 14, 2019-2027, doi:10.2147/DMSO.S307815. 